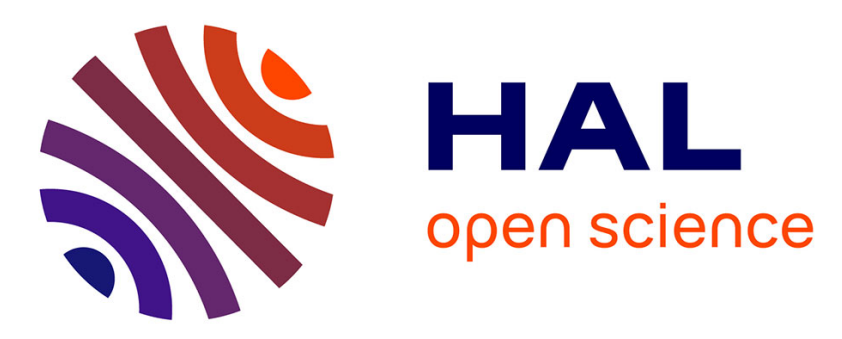

\title{
A Facile and High Yielding Synthesis of Symmetrical and Unsymmetrical Diarylalkynes Using Diethyl Dichloromethylphosphonate as Precursor
}

Virginie Mouriès, Rachel Waschbüsch, John Carran, Philippe Savignac

\section{To cite this version:}

Virginie Mouriès, Rachel Waschbüsch, John Carran, Philippe Savignac. A Facile and High Yielding Synthesis of Symmetrical and Unsymmetrical Diarylalkynes Using Diethyl Dichloromethylphosphonate as Precursor. Synthesis: Journal of Synthetic Organic Chemistry, 1998, 1998 (3), pp.271-274. 10.1055/s-1998-2037 . hal-03166314

\author{
HAL Id: hal-03166314 \\ https://hal.science/hal-03166314
}

Submitted on 11 Mar 2021

HAL is a multi-disciplinary open access archive for the deposit and dissemination of scientific research documents, whether they are published or not. The documents may come from teaching and research institutions in France or abroad, or from public or private research centers.
L'archive ouverte pluridisciplinaire HAL, est destinée au dépôt et à la diffusion de documents scientifiques de niveau recherche, publiés ou non, émanant des établissements d'enseignement et de recherche français ou étrangers, des laboratoires publics ou privés. 


\title{
A Facile and High Yielding Synthesis of Symmetrical and Unsymmetrical Diarylalkynes Using Diethyl Dichloromethylphosphonate as Precursor
}

\author{
Virginie Mouriès, Rachel Waschbüsch, John Carran, and Philippe Savignac \\ Hétéroéléments et Coordination, URA CNRS 1499, DCPH, Ecole Polytechnique, \\ 91128 Palaiseau Cedex. Fax +33 16933 39 90; e-mail dcph@poly.polytechnique.fr
}

The reaction, under internal quench conditions, of diethyl dichloromethylphosphonate and symmetrical or unsymmetrical diarylketones in the presence of $n$-BuLi, leads to the corresponding symmetrical or unsymmetrical diarylalkynes via a Fritsch-Buttenberg-Wiechell rearrangement in a simple, high yielding, onepot reaction.

We recently reported an efficient synthesis of dichloromethylphosphonate $(\mathbf{1})^{1}$ which is known as a useful precursor for the preparation of dichloroalkenes from carbonyl compounds on large scale..$^{2-5}$

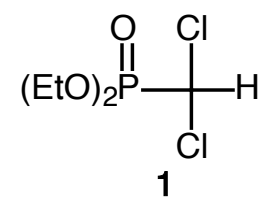

As a component of another project we required preparative access to symmetrical or unsymmetrical diarylalkynes for [4+2] cycloaddition reactions. There are several methods reported in the literature 6 for the synthesis of these type of alkynes and we will just mention the more relevant one. Diarylalkynes are currently prepared via an oxidative coupling between aryl or substituted arylhalides and cuprous acetylides in pyridine ${ }^{7-9}$ or with terminal alkynes catalyzed by palladium at elevated temperatures. ${ }^{10,11}$ In the presence of cuprous iodide, the palladium-catalysed reaction proceeds under mild conditions and gives the diarylalkynes in good yield. ${ }^{12}$ This methodology has been widely used in the preparation of a variety of functionalised diarylalkynes.11-16 Another approach uses the formation of diarylalkenes obtained by a Wittig-Horner reaction from arylmethylphosphonate esters and the corresponding aldehydes. After bromination of the alkenes and an elimination, effected with a base, the diarylalkynes are isolated. A recent preparation of dipyridylalkynes uses this process. ${ }^{17}$ In the same way, 1-chloro-1-arylmethylphosphonate has been introduced as an efficient reagent for the synthesis of phenylpyridylalkynes. ${ }^{18,19}$ It is known that alkynes can equally be obtained through the Fritsch-Buttenberg-Wiechell rearrangement (1894) with 1,1dibromoalkenes as starting materials. ${ }^{20-22}$ The reaction proceeds through the formation of an alkylidene carbene intermediate produced by an $\alpha$-elimination of the 1,1-dibromoalkene 
(Scheme 1). However, as recently reported, the reaction is frequently non-specific, giving mixtures of compounds. ${ }^{23-25}$

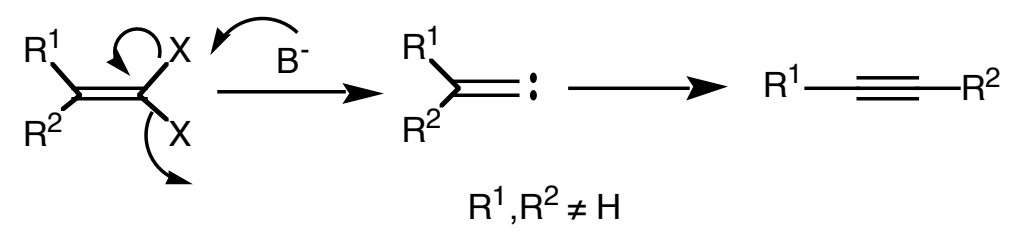

\section{Scheme 1}

We wish to report the generation of symmetrical and unsymmetrical diarylalkynes in a onepot synthesis from 1 and symmetrical or unsymmetrical diarylketones in a FritschButtenberg-Wiechell rearrangement (Scheme 2). As previously described in our synthesis of chloroalkynes and terminal alkynes ${ }^{1}$ we use internal quench conditions. Simultaneous addition of $\mathbf{1}$ and the prospective ketone (2) to LiHMDS in THF solution at low temperatures leads to a phosphorylated carbanion which is only present for a short duration before it reacts with the ketone (2) to give the non-isolated gem-dichloroalkene (3). These conditions thus eliminate the possibility of decomposition of the phosphorylated carbanion to unwanted products. The reactions are easily monitored by ${ }^{31} \mathrm{P}$ NMR spectroscopy as on warming to room temperature the end of the first stage of the reaction is signalled by a single lithiophosphate peak ( ${ }^{31} \mathrm{P}$ NMR $\left.\delta(\mathrm{THF})+0.4 \mathrm{ppm}\right)$. Next, the reaction medium is cooled to $60^{\circ} \mathrm{C}$ and two equivalents of $n$-BuLi are added followed by slow (40 min) warming to $0^{\circ} \mathrm{C}$. This procedure gives the carbene (4) which rearranges to give the required products (5). Occasionaly the reaction mixture is observed to have a brown colouration but this does not affect the result of the experiment. Neutral hydrolysis followed by ethereal extraction and subsequent drying, filtration and evaporation leads to a crude product which is practically free from impurities as observed by ${ }^{1} \mathrm{H}$ and ${ }^{13} \mathrm{C}$ NMR spectroscopy. Filtration of a hexane solution of the alkyne through a silica plug gives the pure title compounds, in cases where the alkyne is not soluble in this solvent then trituration with hexane yields the pure product. We first attempted the reaction using benzophenone and obtained a 95\% yield of diphenylalkyne 5a on preparative scale, free of any side products. The success achieved in the FritschButtenberg-Wiechell rearrangement of $\mathbf{1}$ with benzophenone prompted us to investigate the reaction of 1 with a large variety of symmetrical and unsymmetrical diarylketones and to study the scope and limitations of the procedure. All the ketones used are commercially available or prepared according to known methods. 


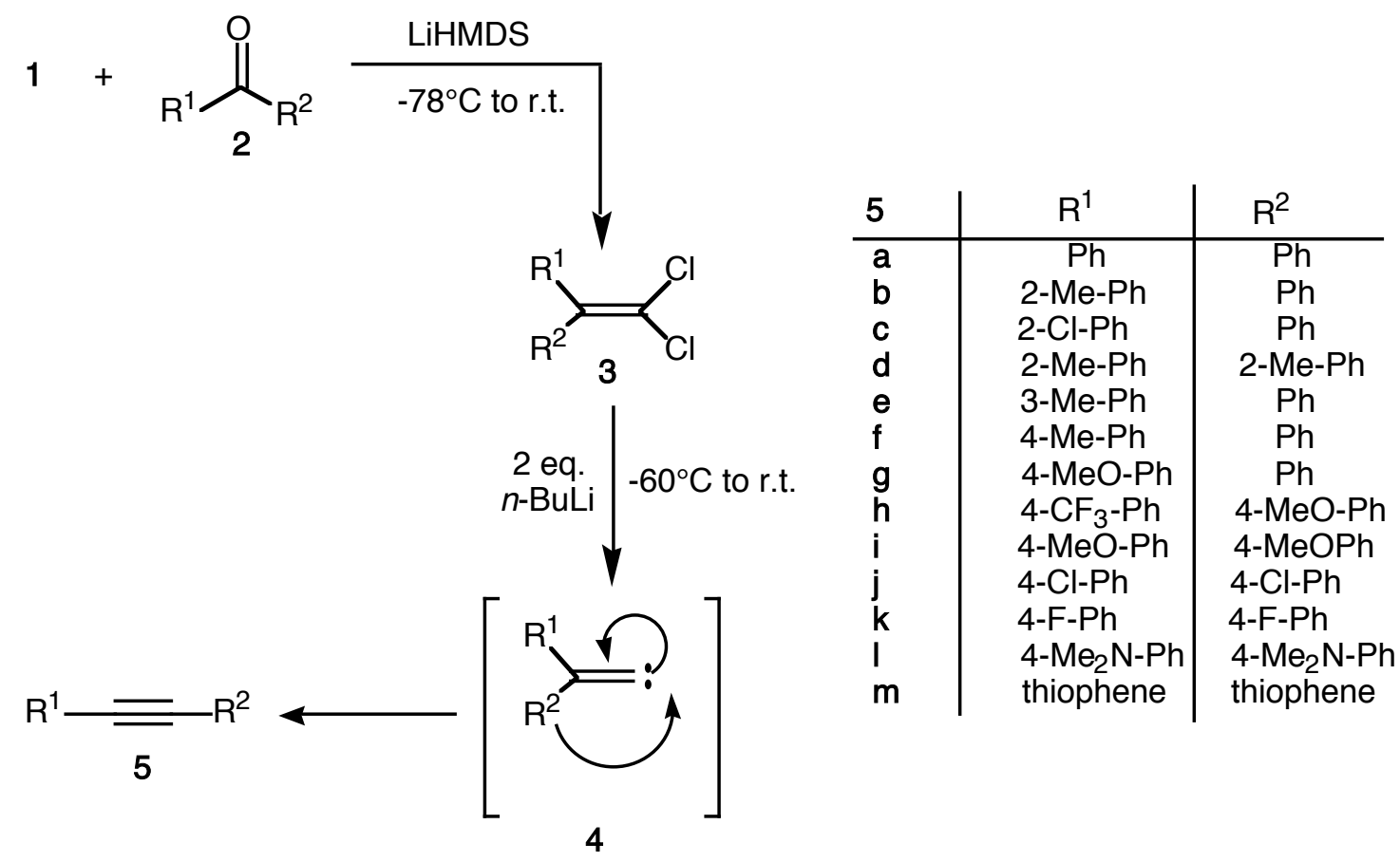

\section{Scheme 2}

It was of interest to explore the effect of the steric bulk of the ketone in the reaction. This was demonstrated by experiments with three ketones of decreasing steric bulk. Thus, 2,2',4,4',6,6'-hexamethylbenzophenone and 2,4,6-trimethylbenzophenone do not react at all however 2,2'-dimethylbenzophenone reacts completely to give the corresponding alkyne $\mathbf{5 d}$ in good yield.

We verified that treatment of $\mathbf{3}$ with excess of $n$-BuLi (2 eq.) was necessary in the second step of the reaction to form the acetylenes 5. In the presence of only one equivalent of $n$-BuLi, this reacts at first with the HMDS present from the first step to reform LiHMDS in preference to a halogen exchange reaction, despite the presence of the two chlorine atoms. The gemdichloroalkenes can be recovered in quantitative yield in this case.

Table 1. Alkynes 5

\begin{tabular}{|c|c|c|c|c|c|}
\hline $\begin{array}{c}\text { Product } \\
\text { ref. }\end{array}$ & $\begin{array}{l}\text { Yield } \\
(\%)\end{array}$ & $\begin{array}{l}\mathrm{mp} \\
\left({ }^{\circ} \mathrm{C}\right)\end{array}$ & $\begin{array}{l}{ }^{1} \mathrm{H} \mathrm{NMR} \quad\left(250 \mathrm{MHz}, \mathrm{CDCl}_{3}\right): \\
\delta, J(\mathrm{~Hz})\end{array}$ & ${ }^{13} \mathrm{C} \mathrm{NMR}\left(250 \mathrm{MHz}, \mathrm{CDCl}_{3}\right): \delta$ & $\begin{array}{l}\mathrm{m} / \mathrm{z}(\mathrm{FAB}) \\
\mathrm{M}^{+}\end{array}$ \\
\hline $5 \mathbf{a}^{8}$ & 95 & $57-59$ & $\begin{array}{l}\text { 7.3-7.4 (m, 3H, Ar-H), 7.42-7.6 } \\
(\mathrm{m}, 2 \mathrm{H}, \mathrm{Ar}-\mathrm{H}) .\end{array}$ & $\begin{array}{l}90.04 \text { ( (C), } 123.91 \text { (Ar-C), } 128.93 \text { (Ar- } \\
\text { C), } 129.00 \text { (Ar-C), 132.28 (Ar-C) }\end{array}$ & 178 \\
\hline $5 \mathbf{b}^{26}$ & 89 & oil & $\begin{array}{l}2.68\left(\mathrm{~s}, 3 \mathrm{H}, \mathrm{Ar}-\mathrm{CH}_{3}\right), 7.23-7.4 \\
(\mathrm{~m}, 3 \mathrm{H}, \mathrm{Ar}-\mathrm{H}), 7.42-7.55(\mathrm{~m}, \\
3 \mathrm{H}, \mathrm{Ar}-\mathrm{H}), 7.61-7.73(\mathrm{~m}, 3 \mathrm{H}, \\
\text { Ar-H) }\end{array}$ & $\begin{array}{l}21.3\left(\mathrm{CH}_{3}\right), 89.04\left(\int \mathrm{C}\right), 94.05\left(\int \mathrm{C}\right), \\
123.63(\mathrm{Ar}-\mathrm{C}), 124.15(\mathrm{Ar}-\mathrm{C}), 126.21 \\
(\mathrm{Ar}-\mathrm{C}), 128.76(\mathrm{Ar}-\mathrm{C}), 128.94(\mathrm{Ar}-\mathrm{C}), \\
130.07(\mathrm{Ar}-\mathrm{C}), 132.1(\mathrm{Ar}-\mathrm{C}), 132.43 \\
(\mathrm{Ar}-\mathrm{C}), 140.71(\mathrm{Ar}-\mathrm{C})\end{array}$ & 192 \\
\hline
\end{tabular}




\begin{tabular}{|c|c|c|c|c|c|}
\hline $5 c^{27}$ & 92 & oil & $\begin{array}{l}7.2-7.33(\mathrm{~m}, 3 \mathrm{H}, \mathrm{Ar}-\mathrm{H}), 7.35- \\
7.5(\mathrm{~m}, 3 \mathrm{H}, \mathrm{Ar}-\mathrm{H}), 7.56-7.65 \\
(\mathrm{~m}, 3 \mathrm{H}, \mathrm{Ar}-\mathrm{H})\end{array}$ & 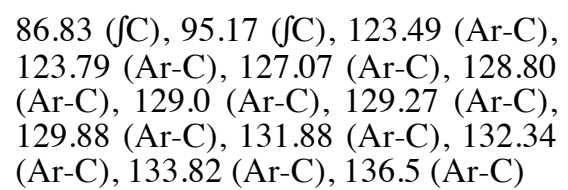 & $212+214$ \\
\hline $5 d^{28}$ & 75 & oil & $\begin{array}{l}2.53\left(\mathrm{~s}, 6 \mathrm{H}, \mathrm{Ar}-\mathrm{CH}_{3}\right), 7.1-7.3 \\
(\mathrm{~m}, 6 \mathrm{H}, \mathrm{Ar}-\mathrm{H}), 7.45-7.55(\mathrm{~m}, \\
2 \mathrm{H}, \mathrm{Ar}-\mathrm{H})\end{array}$ & $\begin{array}{l}21.58\left(\mathrm{CH}_{3}\right), 92.98(\mathrm{SC}), 123.97(\mathrm{Ar}- \\
\mathrm{C}), 126.23(\mathrm{Ar}-\mathrm{C}), 128.83(\mathrm{Ar}-\mathrm{C}), \\
130.1(\mathrm{Ar}-\mathrm{C}), 132.49(\mathrm{Ar}-\mathrm{C}), 140.54 \\
(\mathrm{Ar}-\mathrm{C}),\end{array}$ & 206 \\
\hline $5 e^{29}$ & 91 & $29-30$ & $\begin{array}{l}2.5\left(\mathrm{~s}, 3 \mathrm{H}, \mathrm{Ar}-\mathrm{CH}_{3}\right), 7.2-7.6 \\
(\mathrm{~m}, 7 \mathrm{H}, \mathrm{Ar}-\mathrm{H}), 7.65-7.8(\mathrm{~m}, \\
2 \mathrm{H}, \mathrm{Ar}-\mathrm{H})\end{array}$ & $\begin{array}{l}21.74\left(\mathrm{CH}_{3}\right), 89.73\left(\int \mathrm{C}\right), 90.30\left(\int \mathrm{C}\right), \\
123.72(\mathrm{Ar}-\mathrm{C}), 124.03(\mathrm{Ar}-\mathrm{C}), 128.72 \\
(\mathrm{Ar}-\mathrm{C}), 128.82(\mathrm{Ar}-\mathrm{C}), 128.90(\mathrm{Ar}-\mathrm{C}), \\
129.30(\mathrm{Ar}-\mathrm{C}), 132.17(\mathrm{Ar}-\mathrm{C}), 132.77 \\
(\mathrm{Ar}-\mathrm{C}), 138.50(\mathrm{Ar}-\mathrm{C})\end{array}$ & 192 \\
\hline $\mathbf{5 f}^{30}$ & 93 & $59-61$ & $\begin{array}{l}2.43\left(\mathrm{~s}, 3 \mathrm{H}, \mathrm{Ar}-\mathrm{CH}_{3}\right), 7.13-7.3 \\
(\mathrm{~m}, 2 \mathrm{H}, \mathrm{Ar}-\mathrm{H}), 7.32-7.7(\mathrm{~m}, \\
7 \mathrm{H}, \mathrm{Ar}-\mathrm{H})\end{array}$ & $\begin{array}{l}22.12\left(\mathrm{CH}_{3}\right), 88.38\left(\int \mathrm{C}\right), 90.22\left(\int \mathrm{C}\right), \\
120.82(\mathrm{Ar}-\mathrm{C}), 121.50(\mathrm{Ar}-\mathrm{C}), 128.69 \\
(\mathrm{Ar}-\mathrm{C}), 128.94(\mathrm{Ar}-\mathrm{C}), 129.09(\mathrm{Ar}-\mathrm{C}), \\
129.74(\mathrm{Ar}-\mathrm{C}), 132.14(\mathrm{Ar}-\mathrm{C}), 138.98 \\
(\mathrm{Ar}-\mathrm{C})\end{array}$ & 192 \\
\hline $5 \mathrm{~g}^{31}$ & 88 & $52-54$ & $\begin{array}{l}3.64\left(\mathrm{~s}, 3 \mathrm{H}, \mathrm{CH}_{3} \mathrm{O}\right), 6.65-6.75 \\
(\mathrm{~m}, 3 \mathrm{H}, \mathrm{Ar}-\mathrm{H}), 7.1-7.25(\mathrm{~m}, \\
3 \mathrm{H}, \mathrm{Ar}-\mathrm{H}), 7.29-7.4(\mathrm{~m}, 3 \mathrm{H}, \\
\mathrm{Ar}-\mathrm{H})\end{array}$ & $\begin{array}{l}55.66\left(\mathrm{OCH}_{3}\right), 88.65\left(\mathrm{C} \int\right), 90.01(\mathrm{C}), \\
114.51(\mathrm{Ar}-\mathrm{C}), 115.84(\mathrm{Ar}-\mathrm{C}), 124.12 \\
(\mathrm{Ar}-\mathrm{C}), 127.82(\mathrm{Ar}-\mathrm{C}), 128.46(\mathrm{Ar}-\mathrm{C}), \\
128.85(\mathrm{Ar}-\mathrm{C}), 131.94(\mathrm{Ar}-\mathrm{C}), 133.56 \\
(\mathrm{Ar}-\mathrm{C}), 160.11\left(\mathrm{Ar}-\mathrm{OCH}_{3}\right)\end{array}$ & 208 \\
\hline $5 h$ & 97 & $110-112$ & $\begin{array}{l}3.85\left(\mathrm{~s}, 3 \mathrm{H}, \mathrm{CH}_{3} \mathrm{O}\right), 6.8-6.95 \\
(\mathrm{~m}, 2 \mathrm{H}, \mathrm{Ar}-\mathrm{H}), 7.4-7.51(\mathrm{~m}, \\
2 \mathrm{H}, \mathrm{Ar}-\mathrm{H}), 7.59(\mathrm{~s}, 4 \mathrm{H}, \mathrm{Ar}-\mathrm{H})\end{array}$ & $\begin{array}{l}56.01\left(\mathrm{OCH}_{3}\right), 87.53(\mathrm{fC}), 92.60(\mathrm{fC}), \\
114.8 \text { (Ar-C), } 125.9 \text { (Ar-C), } 127.4- \\
131.9(3 \mathrm{x} \mathrm{Ar}-\mathrm{C}), 132.3 \mathrm{Ar}-\mathrm{C}), 133.9 \\
(\mathrm{Ar}-\mathrm{C}), 160.7 \text { (Ar-C). }\end{array}$ & 276 \\
\hline $\mathbf{5 i}^{32}$ & 86 & $150-152$ & $\begin{array}{l}3.85(\mathrm{~s}, 6 \mathrm{H}, \mathrm{MeO}), 6.82-6.92 \\
(\mathrm{~m}, 4 \mathrm{H}, \mathrm{Ar}-\mathrm{H}), 7.41-7.5(\mathrm{~m}, \\
4 \mathrm{H}, \mathrm{Ar}-\mathrm{H})\end{array}$ & $\begin{array}{l}55.81\left(\mathrm{OCH}_{3}\right), 88.59(\mathrm{C}), 114.57(\mathrm{Ar}- \\
\mathrm{C}), 116.30(\mathrm{Ar}-\mathrm{C}), 133.49(\mathrm{Ar}-\mathrm{C}), \\
160.0(\mathrm{Ar}-\mathrm{C})\end{array}$ & 238 \\
\hline $\mathbf{5 j}^{33}$ & 86 & $174-176$ & $\begin{array}{l}\text { 7.31-7.41 (m, 4H, Ar-H), 7.43- } \\
7.54(\mathrm{~m}, 4 \mathrm{H}, \mathrm{Ar}-\mathrm{H})\end{array}$ & $\begin{array}{l}89.83\left(\int \mathrm{C}\right), 122.09 \text { (Ar-C), } 129.41(\mathrm{Ar}- \\
\mathrm{C}), 133.45(\mathrm{Ar}-\mathrm{C}), 135.16(\mathrm{Ar}-\mathrm{C})\end{array}$ & $246+248$ \\
\hline $\begin{array}{l}\mathbf{5 k}^{33,3} \\
4\end{array}$ & 89 & $94-96$ & $\begin{array}{l}\text { 6.9-7.15 (m, 4H, Ar-H), 7.43- } \\
7.6(\mathrm{~m}, 4 \mathrm{H}, \text { Ar-H })\end{array}$ & $\begin{array}{l}88.65(\mathrm{C}), 116.33\left(\mathrm{~d},{ }^{2} \mathrm{~J}_{\mathrm{CF}}=22, \mathrm{Ar}-\mathrm{C}\right) \\
119.9(\mathrm{Ar}-\mathrm{C}), 134.12\left(\mathrm{~d},{ }^{3} \mathrm{~J}_{\mathrm{CF}}=8, \mathrm{Ar}-\right. \\
\mathrm{C}), 162.5\left(\mathrm{~d},{ }^{1} \mathrm{~J}_{\mathrm{CF}}=250, \mathrm{Ar}-\mathrm{C}\right)\end{array}$ & 214 \\
\hline $5 \mathbf{l}^{35}$ & 87 & $240-243$ & $\begin{array}{l}3.02\left(\mathrm{~s}, 12 \mathrm{H}, \mathrm{N}\left(\mathrm{CH}_{3}\right)_{2}\right), 6.65 \\
(\mathrm{~d}, 4 \mathrm{H}, J=8.5, \mathrm{Ar}-\mathrm{H}), 7.4(\mathrm{~d}, \\
4 \mathrm{H}, J=8.5, \mathrm{Ar}-\mathrm{H})\end{array}$ & $\begin{array}{l}40.95\left(\mathrm{CH}_{3}\right), 88.76(\mathrm{fC}), 111.78(\mathrm{Ar}- \\
\mathrm{C}), 112.60(\mathrm{Ar}-\mathrm{C}), 133.03(\mathrm{Ar}-\mathrm{C}), \\
150.35(\mathrm{Ar}-\mathrm{C})\end{array}$ & 264 \\
\hline $\mathbf{5} \mathbf{m}^{28,3}$ & 95 & $66-70$ & $\begin{array}{l}6.99(\mathrm{dd}, 2 \mathrm{H}, J=5.1,3.7 \\
\left.\mathrm{C}_{4} \mathrm{H}_{3} \mathrm{~S}\right), 7.26(\mathrm{~d}, 2 \mathrm{H}, J=3.7 \\
\left.\mathrm{C}_{4} \mathrm{H}_{3} \mathrm{~S}\right), 7.25-7.28 \quad(\mathrm{~m}, \quad 2 \mathrm{H}, \\
\left.\mathrm{C}_{4} \mathrm{H}_{3} \mathrm{~S}\right)\end{array}$ & $\begin{array}{l}86.9 \quad\left(\int \mathrm{C}\right), \quad 127.8 \quad\left(\mathrm{C}_{4} \mathrm{H}_{3} \mathrm{~S}\right), \\
\left(\mathrm{C}_{4} \mathrm{H}_{3} \mathrm{~S}\right), 128.3\left(\mathrm{C}_{4} \mathrm{H}_{3} \mathrm{~S}\right), 130.4-130.5 \\
\left(\mathrm{C}_{4} \mathrm{H}_{3} \mathrm{~S}\right), 132.8\left(\mathrm{C}_{4} \mathrm{H}_{3} \mathrm{~S}\right)\end{array}$ & 190 \\
\hline
\end{tabular}

We also established that at low temperature, the second step of the reaction involving the halogen-metal exchange is fast whereas the formation of carbene is slow. Performing the reaction using benzophenone as ketone we obtained gem-dichloroolefin $\mathbf{3}$ as usual, then addition of $n$-BuLi (2 eq.) gives, after $45 \mathrm{~min}$ at $-80^{\circ} \mathrm{C}$, a $60: 40$ ratio of vinyl (6) to acetylenic product (5a) after ethanolysis. With 4,4'-dichlorobenzophenone addition of $n$-BuLi gives, after $5 \mathrm{~min}$ at $-85^{\circ} \mathrm{C}$, a $87: 13$ ratio (vinyl to acetylenic). Finally, after the addition of $n$-BuLi we maintained the temperature at $-90^{\circ} \mathrm{C}$ for $5 \mathrm{~min}$ before ethanolysis and obtained, after normal workup, the gem-diarylmonochloroolefins 6 (in 50 / $50 \mathrm{E}+\mathrm{Z}$ mixture in the case of $\mathbf{5 f}$ ) described in Table 2 as the sole products. Thus the formation of the carbene intermediate 4 is a slow process which occurs at temperatures higher than $-80^{\circ} \mathrm{C}$. This 
experimental evidence is also aided by the observation that at $-90^{\circ} \mathrm{C}$ after $n$-BuLi addition, the reaction mixture remains colourless.

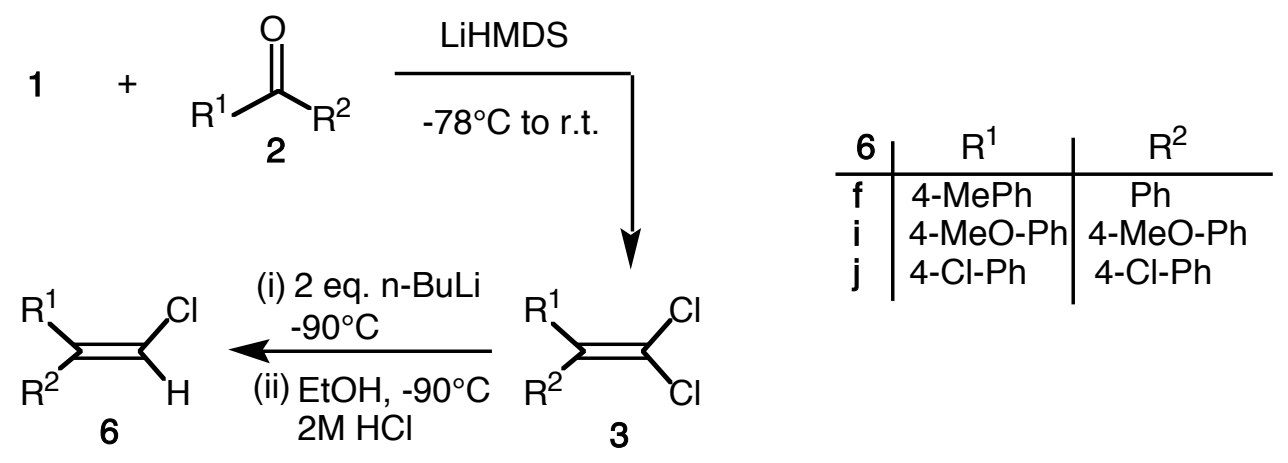

\section{Scheme 3}

Table 2. Chloroolefins 6

\begin{tabular}{|c|c|c|c|c|c|}
\hline $\begin{array}{c}\text { Produc } 1 \\
\text { Ref. }\end{array}$ & $\begin{array}{l}\text { Yield } \\
(\%)\end{array}$ & $\begin{array}{l}\mathrm{mp} \\
\left({ }^{\circ} \mathrm{C}\right)\end{array}$ & $\begin{array}{l}{ }^{1} \mathrm{H} \mathrm{NMR}\left(250 \mathrm{MHz}, \mathrm{CDCl}_{3}\right): \\
\delta, J(\mathrm{~Hz})\end{array}$ & ${ }^{13} \mathrm{C} \mathrm{NMR}\left(250 \mathrm{MHz}, \mathrm{CDCl}_{3}\right): \delta$ & $\begin{array}{l}\mathrm{m} / \mathrm{z}(\mathrm{FAB}) \\
\mathrm{M}^{+}\end{array}$ \\
\hline $\mathbf{6 f}^{36}$ & 84 & oil & $\begin{array}{l}2.4\left(\mathrm{~s}, 3 \mathrm{H}, \mathrm{ArCH}_{3}\right), 2.45(\mathrm{~s}, 3 \mathrm{H}, \\
\left.\mathrm{ArCH}_{3}\right), 6.55(\mathrm{~m}, 1 \mathrm{H},=\mathrm{CHCl}), \\
7.18(\mathrm{~s}, 2 \mathrm{H}, \mathrm{Ar}-\mathrm{H}), 7.28-7.52 \\
(\mathrm{~m}, 5 \mathrm{H}, \mathrm{Ar}-\mathrm{H}), 7.29(\mathrm{~s}, 2 \mathrm{H}, \mathrm{Ar}- \\
\mathrm{H})\end{array}$ & $\begin{array}{l}21.7,21.9\left(\mathrm{CH}_{3}\right), 115.7(=\mathrm{C}), 116.1 \\
(=\mathrm{C}), 128.2,128.4 \quad(\mathrm{Ar}-\mathrm{C}), 128.5, \\
128.6(\mathrm{Ar}-\mathrm{C}), 128.8,129.0(\mathrm{Ar}-\mathrm{C}), \\
129.5,129.7(\mathrm{Ar}-\mathrm{C}), 130.4,130.5(\mathrm{Ar}- \\
\mathrm{C}), 135.2,137.9(\mathrm{Ar}-\mathrm{C}), 138.3,138.5 \\
(\mathrm{Ar}-\mathrm{C}), 141.0,144.4(\mathrm{Ar}-\mathrm{C})\end{array}$ & $228+230$ \\
\hline $6 \mathbf{i}^{21}$ & 89 & $72-73$ & $\begin{array}{l}3.8-3.85(\mathrm{~m}, 3 \mathrm{H},), 6.45(\mathrm{~s}, 1 \mathrm{H}, \\
=\mathrm{CHCl}), 6.79-6.98(\mathrm{~m}, 4 \mathrm{H}, \mathrm{Ar}- \\
\mathrm{H}), 7.08-7.32(\mathrm{~m}, 4 \mathrm{H}, \mathrm{Ar}-\mathrm{H})\end{array}$ & $\begin{array}{l}55.82\left(\mathrm{CH}_{3} \mathrm{O}\right), 114.09(=\mathrm{C}), 114.33 \\
(=\mathrm{C}), 128.94 \text { (Ar-C), } 129.64(\mathrm{Ar}-\mathrm{C}), \\
130.58(\mathrm{Ar}-\mathrm{C}), 131.64(\mathrm{Ar}-\mathrm{C}), 131.85 \\
(\mathrm{Ar}-\mathrm{C}), 133.67(\mathrm{Ar}-\mathrm{C}), 143.47(\mathrm{Ar}-\mathrm{C}), \\
159.75(\mathrm{Ar}-\mathrm{C}), 160.12(\mathrm{Ar}-\mathrm{C})\end{array}$ & 276 \\
\hline $\mathbf{6 j}^{31 \mathrm{a}}$ & 87 & $65-67$ & $\begin{array}{l}6.58(\mathrm{~s}, 1 \mathrm{H},=\mathrm{CHCl}), 7.08-7.18 \\
(\mathrm{~m}, 2 \mathrm{H}, \mathrm{Ar}-\mathrm{H}), 7.2-7.3(\mathrm{~m}, 4 \mathrm{H}, \\
\mathrm{Ar}-\mathrm{H}), 7.33-7.4(\mathrm{~m}, 2 \mathrm{H}, \mathrm{Ar}-\mathrm{H})\end{array}$ & $\begin{array}{l}117.41(=\mathrm{C}), 117.48(=\mathrm{C}), 129.23(\mathrm{Ar}- \\
\mathrm{C}), 129.35(\mathrm{Ar}-\mathrm{C}), 129.55 \text { (Ar-C), } \\
131.85(\mathrm{Ar}-\mathrm{C}), 134.74(\mathrm{Ar}-\mathrm{C}), 134.92 \\
(\mathrm{Ar}-\mathrm{C}), 136.03(\mathrm{Ar}-\mathrm{C}), 138.73 \text { (Ar-C), } \\
142.33(\mathrm{Ar}-\mathrm{C})\end{array}$ & $\begin{array}{r}282+284 \\
+286+288\end{array}$ \\
\hline
\end{tabular}

In conclusion we have presented in this paper a useful method for the preparation of symmetrical or unsymmetrical diarylalkynes $\mathbf{5}$. This facile, one-pot reaction starting from the versatile reagent $\mathbf{1}$ works well with a wide range of diaromatic ketones and a variety of substituents are tolerated under the reaction conditions. The simplicity of the experimental procedure, and the high yields obtained make this approach attractive. With a small variation in reaction conditions a variety of monochloroolefins 6 have been prepared.

NMR spectra were recorded on a Bruker AC 200 spectrometer operating at $200 \mathrm{MHz}$ for proton, $50.3 \mathrm{MHz}$ for carbon and $81.01 \mathrm{MHz}$ for phosphorus. ${ }^{31} \mathrm{P}$ downfield shifts $(\delta)$ are expressed with a positive sign, in $\mathrm{ppm}$, relative to external $85 \% \mathrm{H}_{3} \mathrm{PO}_{4}$ in $\mathrm{H}_{2} \mathrm{O}$. ${ }^{1} \mathrm{H}$ and ${ }^{13} \mathrm{C}$ chemical shifts $(\delta)$ are reported in ppm relative to $\mathrm{CDCl}_{3}$ as internal standard. Coupling constants $(J)$ are given in Hz. The following abbreviations are used: s, d, t, q, m 
for singlet, doublet, triplet, quadruplet and multiplet respectively. Organic solvents were purified by standard procedures. THF was distilled under an inert atmosphere from purple solutions of sodium-benzophenone ketyl. The synthesis of all compounds was carried out under dry nitrogen.

\section{Synthesis of diarylalkynes 5; General procedure:}

$n$-BuLi (14 $\mathrm{ml}$ of $1.6 \mathrm{M}$ solution in hexane, $22 \mathrm{mmol})$ is added to THF $(20 \mathrm{ml})$ and cooled to $-78{ }^{\circ} \mathrm{C}$. A solution of HMDS (3.90 g; $24 \mathrm{mmol})$ in THF $(20 \mathrm{ml})$ is then slowly added at this temperature via a dropping funnel. The reaction mixture is then allowed to slowly warm to $0^{\circ} \mathrm{C}$ before once again cooling to $-78{ }^{\circ} \mathrm{C}$. A mixture of diethyl dichloromethylphosphonate $(4.42 \mathrm{~g} ; 22 \mathrm{mmol})$ and the diaryl ketone $(20 \mathrm{mmol})$ in THF $(50 \mathrm{ml})$ is then slowly added to the reaction mixture maintaining the temperature at $-78{ }^{\circ} \mathrm{C}$. Stirring is continued at this temperature for $10 \mathrm{~min}$. and then the reaction is allowed to warm to $0^{\circ} \mathrm{C}$ over a period of $30 \mathrm{~min}$. The reaction mixture is cooled to $-60{ }^{\circ} \mathrm{C}$ and $n$ - $\mathrm{BuLi}(29 \mathrm{ml}, 46 \mathrm{mmol})$ is added. The brownish reaction mixture is then left to warm to $0{ }^{\circ} \mathrm{C}$ over a period of $30 \mathrm{~min} . \mathrm{H}_{2} \mathrm{O}(20 \mathrm{ml})$ is then added to the reaction mixture with rapid stirring and the mixture is extracted with diethyl ether $(3 \times 30 \mathrm{ml})$ and the ethereal extractions are washed with $\mathrm{H}_{2} \mathrm{O}$ $(2 \times 20 \mathrm{ml})$ then with $2 \mathrm{M} \mathrm{HCl}(3 \times 5 \mathrm{ml})$ before drying $\left(\mathrm{MgSO}_{4}\right)$, filtration and evaporation to yield the crude title compounds. These can be purified further by filtration through a silica plug using hexane as eluent or trituration with dry hexane or column chromatography .

\section{Synthesis of gem-diarylchloroolefins 6; General procedure:}

As above except that the second addition of 2 equiv. of $n$ - BuLi is carried out at $-90{ }^{\circ} \mathrm{C}$ and the reaction mixture is kept at this temperature for $5 \mathrm{~min}$ before ethanol $(10 \mathrm{ml})$ is added at $-90{ }^{\circ} \mathrm{C}$. The reaction is then worked up as above.

The authors would like to thank the CNRS for financial support for J. C. and R. W.

(1) (a) Carran, J.; Waschbüsch, R.; Marinetti, A.; Savignac, P. Synthesis 1996, 1494.

(b) Marinetti, A.; Savignac, P. Organic Syntheses 1997, 74, 108.

(c) Waschbüsch, R.; Carran, J.; Marinetti, A.; Savignac, P. Synthesis 1997, 727.

(2) Seyferth, D.; Marmor, R. S. J. Organomet. Chem. 1973, 59, 237.

(3) Savignac, P.; Petrova, J.; Dreux, M.; Coutrot, P. Synthesis 1975, 535.

(4) Pflieger, D.; Muckensturm, B. Tetrahedron 1989, 45, 2031.

(5) Villieras, J.; Perriot, P.; Normant, J. F. Synthesis 1975, 458.

(6) Furber, M. in Comprehensive Org. Funct. Group Transf., A. R. Katritsky et al., Eds, Pergamon, 1995, vol. 1, Chap.1.21, p 997

(7) Castro, C. E.; Stephens, R. D. J. Org. Chem. 1963, 28, 2163.

(8) Stephens, R. D.; Castro, C. E. J. Org. Chem. 1963, 28, 3313.

(9) Castro, C. E.; Havlin, R.; Honwad, V. K.; Malte, A.; Moje, S. J. Am. Chem. Soc. 1969, 91, 6464.

(10) Dieck, H. A.; Heck, F. R. J. Organomet. Chem. 1975, 93, 259. 
(11) Casar, L. J. Organomet. Chem. 1975, 93, 253.

(12) Sonogashira, K.; Tohda, Y.; Hagihara, N. Tetrahedron Lett. 1975, 4467.

(13) Takahashi, S.; Kuroyama, Y.; Sonogashira, K.; Hagihara, N. Synthesis 1980, 627.

(14) Austin, W. B.; Bilow, N.; Kelleghan, W. J.; Lau, K. S. Y. J. Org. Chem. 1981, 46, 2280 .

(15) (a) Ratovelomanana, V.; Linstrumelle, G. Synth. Commun. 1981, 11, 917.

(b) Ratovelomanana, V.; Linstrumelle, G.Tetrahedron Lett. 1984, 25, 6001.

(c) Guillerm, D.; Linstrumelle, G. Tetrahedron Lett. 1986, 27, 5857.

(16) Tao, W.; Nesbitt, S.; Heck, F. R. J. Org. Chem. 1990, 55, 63.

(17) Windscheif, P. M.; Vögtle, F. Synthesis 1994, 87.

(18) Zimmer, H.; Bercz, P. J.; Maltenieks, O. J.; Moore, M. W. J. Am. Chem. Soc. 1965, 87,2777 .

(19) Zimmer, H.; Hickey, K. R.; Schumacher, R. J. Chimia 1974, 28, 656.

(20) Fritsch, P. Liebigs Ann. Chem. 1894, 279, 319.

(21) Buttenberg, W. P. Liebigs Ann. Chem. 1894, 279, 327.

(22) Wiechell, H. Liebigs Ann. Chem. 1894, 279, 337.

(23) Sato, H.; Isono, N.; Miyoshi, I.; Mori, M. Tetrahedron 1996, 52, 8143.

(24) Kunishima, M.; Hioki, K.; Ohara, T.; Tani, S. J. Chem. Soc., Chem. Commun. 1992, 219.

(25) Harada, T.; Hara, D.; Hattori, K.; Oku, A. Tetrahedron Lett. 1988, 29 , 3821.

(26) Merkushev, E. B.; Skorokhodova, T. S. J. Org. Chem. U.S.S.R. (Engl. Transl.) 1982, $18,308$.

(27) Chen, Q.-Y.; Yang, Z.-Y. Tetrahedron Lett. 1986, 27, 1171.

(28) D'Auria, M. Synth. Commun. 1992, 22, 2393.

(29) King, A. O., Negishi, E.; Villani, F. J., Jr.; Silveira, A., Jr. J. Org. Chem. 1978, 43, 358.

(30) Sukhomlinova L. I.; Luzikova E. V.; Tolstaya T. P.; Bumagin N. A.; Beletskaya I. P. Russ. Chem. Bl. (Engl. Transl.) 1995, 44, 769.

(31) (a) Katritzky, A. R.; Boulton, A. J.; Short, D. J. J. Chem. Soc. 1960, 1519.

(b) Martin, S.; Sauvêtre, R.; Normant, J.-F. Tetrahedron Lett. 1982, 23, 4329.

(c) McEwen, W. E.; Guzikowski, A. P.; Wolf, A. P. J. Fluorine Chem. 1984, 25, 169.

(d) Dzhemilev, U. M.; Saraev, R. A.; Vyrypaev, E. M.; Ibragimov, A. G. Bull. Acad. Sci. U.S.S.R. Div. Chem. Sci. (Engl. Transl.) 1987, 36, 1888.

(32) (a) Koebrich, G.; Trapp, H. Chem. Ber. 1966, 99, 680.

(b) Kaufman, R. J.; Sidhu, R. S. J. Org. Chem. 1982, 47, 4941.

(c) Latif, K. A.; Ali, M. Umar Indian J. Chem. Sect. B 1984, 23, 471.

(d) Ramana, D. V.; Krishna, N. V. S. Rama Org. Mass Spectrom. 1989, 24, 903. 
(e) Silverberg, L. J.; Wu, G.; Rheingold, A. L.; Heck, R. F. J. Organomet. Chem. 1991, 409, 411.

(f) Taniguchi, Y.; Fujii, N.; Makioka, Y.; Takaki, K.; Fujiwara, Y. Chem. Lett. 1993, 7 , 1165 .

(g) Weiss, R.; Seubert, J. Angew. Chem. 1994, 106, 900.

(h) Cummins, C. H. Tetrahedron Lett. 1994, 35, 857.

(33) Weis, C. D. Helv. Chim. Acta 1966, 49, 234.

(34) (a) Gascoyne, J. M.; Mitchell, P. J.; Phillips, L. J. Chem. Soc. Perkin Trans. 2 1977, 1051 .

(b) Paventi, M.; Elce, E.; Jackman, R. J., Hay, A. S. Tetrahedron Lett. 1992, 33, 6405.

(35) Inada, A.; Nakamura, Y.; Morita, Y. Chem. Pharm. Bull. 1982, 30, 1041.

(36) Pal, M.; Kundu, N.G. J. Chem. Soc. Perkin Trans. 1 1996, 449.

(37) (a) Koebrich, G.; Trapp, H.; Hornke, I. Chem. Ber. 1967, 100, 961

(b) Suarez, A.R.; Mazzieri, M.R. J. Org. Chem. 1987, 52, 1145 